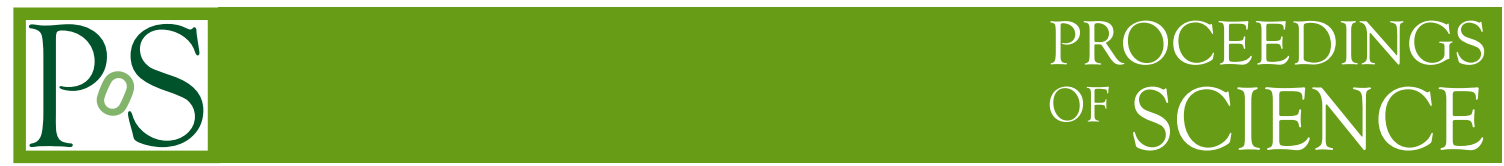

\title{
Dynamical net charge fluctuations at RHIC energies in STAR
}

\author{
Bhanu SHARMA (for the STAR Collaboration)* \\ Panjab University, Chandigarh, India \\ E-mail: bhanu.pu@gmail.com
}

\begin{abstract}
We present preliminary results from the study of dynamical net-charge fluctuations measured in $\mathrm{Au}+\mathrm{Au}$ collisions at $\sqrt{s_{N N}}=39 \mathrm{GeV}$ to $7.7 \mathrm{GeV}$ and compare with the published results at higher collision energies. We also present the centrality dependence of the dynamical net-charge fluctuations.
\end{abstract}

7th International Conference on Physics and Astrophysics of Quark Gluon Plasma

1-5 February, 2015

Kolkata, India

${ }^{*}$ Speaker. 


\section{Introduction}

The STAR experiment at the Relativistic Heavy Ion Collider (RHIC) provides the ability to investigate the behaviour of strongly interacting matter at high density and to study the Quark Gluon Plasma (QGP). In the year 2010 (Run 10), RHIC started its Beam Energy Scan (BES) program and collided $\mathrm{Au}+\mathrm{Au}$ ions from $\sqrt{s_{N N}}=39 \mathrm{GeV}$ down to $7.7 \mathrm{GeV}$ covering $112<\mu_{B}<$ $410 \mathrm{MeV}$. This allows one to access and probe broad regions of the QCD phase diagram. Event-byevent net-charge fluctuations have been proposed as an indicator of the QGP formation in heavy ion collisions. The fluctuation in net-charge depends on the squares of the charges present in the system, which depends on the state from which it originates. The system passing through a QGP phase which has quarks as charge carriers should result in a significantly different net-charge fluctuation as compared to Hadron Gas (HG). The variance of the event-by-event difference of the numbers of positive and negative particles scaled by the total charged particle multiplicity, a quantity called $D$, should be approximately four times smaller in a QGP medium than in a gas of hadrons. The charge fluctuation is measured in terms of $D$ defined as :

$$
D=4 \frac{\left\langle\delta Q^{2}\right\rangle}{\left\langle N_{c h}\right\rangle}
$$

where $\left\langle\delta Q^{2}\right\rangle$ is the net charge variance, $Q=N_{+}-N_{-}$and $N_{c h}=N_{+}+N_{-}$. Here $N_{+}$and $N_{-}$are the number of negative and positive particles, measured in specific transverse momentum $\left(p_{T}\right)$ and pseudorapidity $(\eta)$ window.

The value of $D$ is 4 for an uncorrelated pion gas and is reduced by about $30 \%$ in the presence of resonances. For a thermal system of free quarks and gluons, the value is significantly lower and has been calculated to be $\approx 1$. [1,2]

The event-by-event net-charge fluctuations have also been estimated by calculating the quantity $v_{+-, d y n}$ defined as: $[3,4,5,6]$ :

$$
v_{+-, d y n}=\frac{\left\langle N_{+}\left(N_{+}-1\right)\right\rangle}{\left\langle N_{+}\right\rangle^{2}}+\frac{\left\langle N_{-}\left(N_{-}-1\right)\right\rangle}{\left\langle N_{-}\right\rangle^{2}}-2 \frac{\left\langle N_{-} N_{+}\right\rangle}{\left\langle N_{+}\right\rangle\left\langle N_{-}\right\rangle}
$$

which is a measure of the relative correlation of,++-- and +- pairs. The $v_{+-, d y n}$ has been found to be robust against experimental inefficiencies [7]. The value of $D$ is related to $v_{+-, d y n}$ as :

$$
\left\langle N_{c h}\right\rangle v_{+-, d y n}=D-4
$$

\section{Analysis Details and Results}

The measurements of net-charge fluctuations as a function of centrality in Au+Au collisions at $\sqrt{s_{N N}}=7.7,11.5,19.6,27$ and $39 \mathrm{GeV}$ data collected in years 2010 and 2011 by the STAR experiment are reported. For this analysis, we use charged particle tracks from the Time Projection Chamber (TPC) for $-0.5<\eta<0.5$ with transverse momenta in the range $0.2<p_{T}<5.0 \mathrm{GeV} / c$. For the centrality selection, the uncorrected multiplicity of charged particles within $0.5<|\eta|<$ 1.0 is used in order to avoid the autocorrelation between the centrality definition and the $v_{+-, d y n}$ observable. 


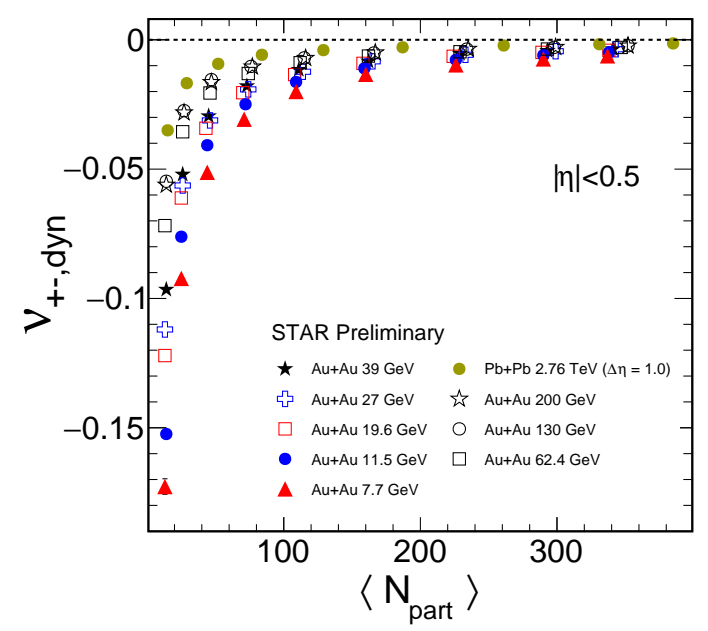

Figure 1: Dynamical net-charge fluctuations, $v_{+-, d y n}$, of charged particles as a function of number of participating nucleons, $\left\langle N_{\text {part }}\right\rangle$.

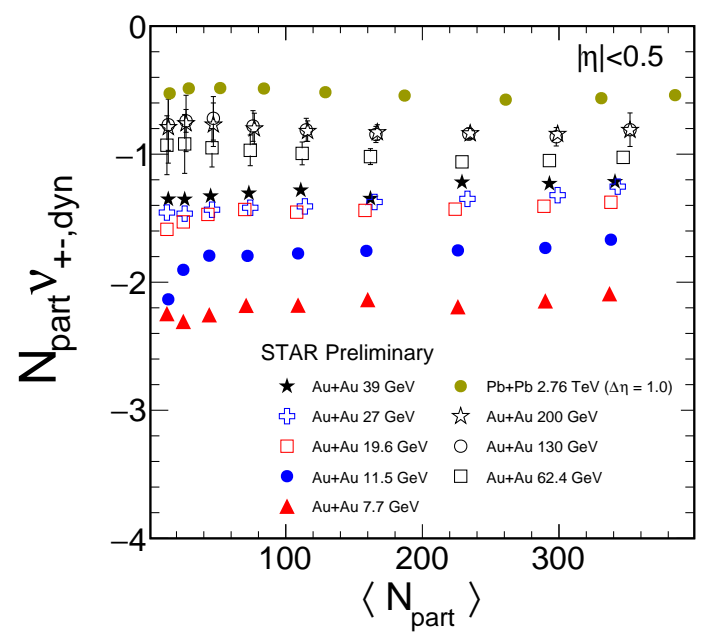

Figure 2: Dynamical net-charge fluctuations, $v_{+-, d y n}$, scaled with the average number of participating nucleons as a function of $\left\langle N_{\text {part }}\right\rangle$.

Figure 1 shows the measurements of $v_{+-, d y n}$ at $\sqrt{s_{N N}}=7.7,11.5,19.6,27$ and $39 \mathrm{GeV}$ as a function of the average number of participating nucleons, $\left\langle N_{\text {part }}\right\rangle$. It also includes the previous results from STAR at $\sqrt{s_{N N}}=200,130$ and $62.4 \mathrm{GeV}$, and ALICE results for $\mathrm{Pb}+\mathrm{Pb}$ collisions at $\sqrt{s_{N N}}=2.76 \mathrm{TeV}[5,8]$. The uncertainties shown are only statistical. In all cases, the values of $v_{+-, d y n}$ are negative indicating the dominance of the correlation between positive and negative charged particles. The strength of the correlation decreases while going from peripheral to central collisions. Also, the magnitude of fluctuations decreases as the beam energy increases. 
Figure 2 shows $v_{+-, d y n}$ scaled with the average number of participating nucleons, $N_{\text {part }} v_{+-, d y n}$, as a function of $\left\langle N_{\text {part }}\right\rangle$. The measured data scaled by the number of participating nucleons exhibits either a weak or no centrality dependence at all of the measured energies.

The magnitude of the net-charge correlations is affected by the global charge conservation and

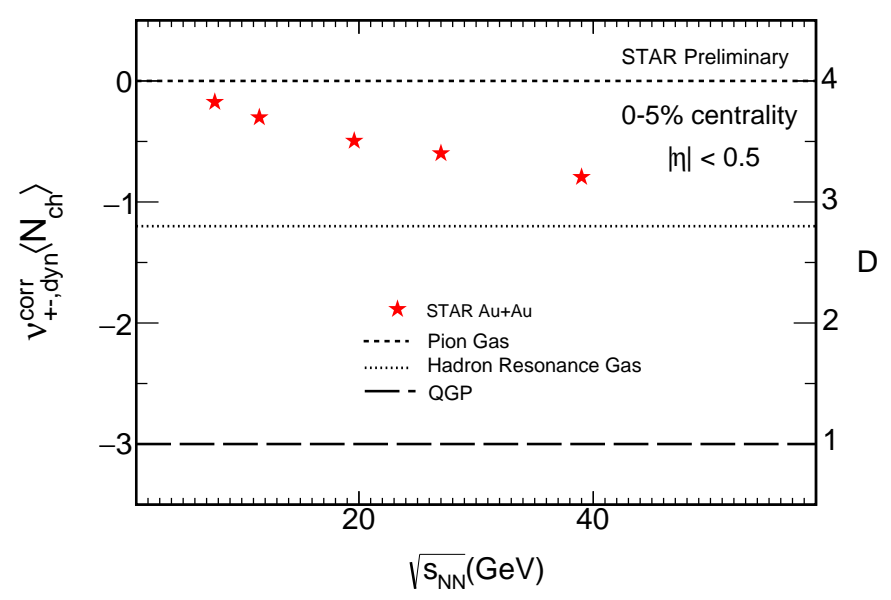

Figure 3: $\left\langle N_{c h}\right\rangle v_{+-, \text {dyn }}^{\text {corr }}$ (left-axis) and $D$ (right-axis) as a function of beam energy for 0-5\% collisions. The theoretical predictions for a Pion Gas, HG and a QGP are also indicated. Systematic uncertainties are not yet included.

the finite size of the colliding system. The contribution of the global charge conservation effect is estimated to be $-4 /\left\langle N_{\text {total }}\right\rangle$, [4] where $\left\langle N_{\text {total }}\right\rangle$ is the average number of charged particles produced over the full phase space. The corrected value of $v_{+-, d y n}$ is calculated as

$$
v_{+-, d y n}^{\text {corr }}=v_{+-, d y n}+\frac{4}{\left\langle N_{\text {total }}\right\rangle}
$$

Here the $\left\langle N_{c h}\right\rangle$ is the efficiency corrected average charged particle multiplicity for $\mathrm{Au}+\mathrm{Au}$ collisions. Both $\left\langle N_{c h}\right\rangle$ and $\left\langle N_{\text {total }}\right\rangle$ have been estimated from the PHOBOS experiment data [9]. Figure 3 shows $\left\langle N_{c h}\right\rangle v_{+-, \text {dyn }}^{\text {corr }}$ along left y-axis and $D$ along right y-axis as a function of the colliding energy for $0-5 \%$ central collisions within $|\eta|<0.5$. The uncertainties shown are statistical only. The theoretical predictions for Pion Gas, Hadron Resonance Gas and QGP have also been displayed in the figure. It can be observed that the net-charge fluctuations corrected for the global charge conservation when scaled with $\left\langle N_{c h}\right\rangle$ decrease with increases in the beam energy. The $D$ measure linearly decreases with increasing energy. The net-charge fluctuations observables approach the expectation for a Pion Gas as the beam energy decreases.

In summary, we report recent results of the net-charge fluctuations for $\mathrm{Au}+\mathrm{Au}$ collisions at 7.7, 11.5, 19.6, 27 and $39 \mathrm{GeV}$. There is a monotonic reduction in the magnitude of dynamical net-charge fluctuations with increasing number of participants. Dynamical net-charge fluctuations are observed to follow approximate $N_{\text {part }}$ scaling. Top 5\% central collisions results show that $\left\langle N_{c h}\right\rangle v_{+-, \text {dyn }}^{\text {corr }}$ generally decreases with increasing colliding energy. 


\section{References}

[1] S. Jeon, V. Koch, Phys. Rev. Lett. 83, 5435 (1999).

[2] S. Jeon, V. Koch, Phys. Rev. Lett. 85, 2076 (2000).

[3] J. Adams et al. [STAR Collaboration] Phys. Rev. C 68, 044905 (2003).

[4] C. Pruneau, S. Gavin, S. Voloshin, Phys. Rev. C 66, 044904 (2002).

[5] B.I. Abelev et al. [STAR Collaboration] Phys. Rev. C 79,024906 (2009).

[6] Bhanu Sharma, Madan M. Aggarwal, Nihar Ranjan Sahoo and Tapan K. Nayak, Phys. Rev. C. 91, 024909 (2015)

[7] J. Nystrand, E. Stenlund, H. Tydesjo, Phys. Rev. C68, 034902 (2003).

[8] B. Abelev et al. (ALICE Collaboration), Phys. Rev. Lett. 110, 152301 (2013).

[9] B. Adler et al. (PHOBOS Collaboration), Phys. Rev. C 83024913 (2011). 